\title{
Different protein composition of low-calorie diet differently impacts adipokine profile irrespective of weight loss in overweight and obese women
}

\author{
R. Mateo-Gallego ${ }^{\text {a,* }}$, I. Lamiquiz-Moneo ${ }^{a}$, S. Perez-Calahorra ${ }^{\text {a }}$, V. Marco-Benedí a \\ A.M. Bea ${ }^{\text {a }}$, L. Baila-Rueda ${ }^{\text {a }}$, M. Laclaustra ${ }^{a}$, J.L. Peñalvo ${ }^{\text {b }}$, F. Civeira ${ }^{a}$, A. Cenarro ${ }^{a}$ \\ ${ }^{a}$ Unidad Clínica y de Investigación en Lípidos y Arteriosclerosis, Hospital Universitario Miguel Servet, Instituto de Investigación Sanitaria Aragón \\ (IIS Aragón), Universidad de Zaragoza, CIBERCV, Zaragoza, Spain \\ ${ }^{b}$ Tufts Friedman School of Nutrition Science E Policy, Boston, MA, USA
}

Received 19 May 2017; received in revised form 27 October 2017; accepted 30 October 2017

Handling Editor: Peter Clifton

Available online

\section{KEYWORDS \\ Protein; \\ Weight loss; \\ Adipokines; \\ Adipose tissue; \\ Obesity; \\ Overweight}

\begin{abstract}
Background and aims: High-protein (HP) diets have shown benefits in cardiometabolic markers such as insulin or triglycerides but the responsible mechanisms are not known. We aimed to assess the effect of three energy-restricted diets with different protein contents $(20 \%$, $27 \%$, and $35 \% ; \sim 80 \%$ coming from animal source) on plasma adipokine concentration and its association with changes in cardiometabolic markers.

Methods: Seventy-six women (BMI $32.8 \pm 2.93$ ) were randomized to one of three caloriereduced diets, with protein, $20 \%, 27 \%$, or $35 \%$; carbohydrates, $50 \%, 43 \%$, or $35 \%$; and fat, $30 \%$, for 3 months. Plasma adipokine (leptin, resistin, adiponectin, and retinol-binding protein 4; RBP4) levels were assessed.

Results: After 3 months, leptin concentration decreased in all groups without differences among them, while resistin levels remained unchanged. Adiponectin concentration heterogeneously changed in all groups ( $P$ for trend $=0.165$ ) and resistin concentration did not significantly change. RPB4 significantly decreased by $-17.5 \%(-31.7,-3.22)$ in $35 \%$-protein diet $(P$ for trend $=0.024$ among diets). Triglycerides improved in women following the $35 \%$-protein diet regardless of weight loss; RBP4 variation significantly influenced triglyceride concentration change by $24.9 \%$ and $25.9 \%$ when comparing $27 \%$ - and $35 \%$ - with $20 \%$-protein diet, respectively. Conclusions: A 35\%-protein diet induced a decrease in RBP4 regardless of weight loss, which was directly associated with triglyceride concentration improvement. These findings suggest that HP diets improve the cardiometabolic profile, at least in part, through changes in adipokine secretion. Whether this beneficial effect of HP diet is due to improvements in hepatic or adipose tissue functionality should be elucidated.

Clinical trial registration: The clinical trial has been registered in ClinicalTrials.gov (Identifier: NCT02160496).

๑ 2017 The Italian Society of Diabetology, the Italian Society for the Study of Atherosclerosis, the Italian Society of Human Nutrition, and the Department of Clinical Medicine and Surgery, Federico II University. Published by Elsevier B.V. All rights reserved.
\end{abstract}

Abbreviations: AgRP, Agouti-related peptide; BMI, Body mass index; CRP, C-reactive protein; GGT, Gamma-glutamyl transpeptidase; GPT, Glutamic-pyruvic transaminase; HbA1c, Glycated hemoglobin; HP, High protein; NPY, Neuropeptide Y; RBP4, Retinol-binding protein 4.

* Corresponding author. Unidad Clínica y de Investigación en Lípidos y Arteriosclerosis, Hospital Universitario Miguel Servet, Av. Isabel La Católica, 1-3, 50009 Zaragoza, Spain. Fax: +34 976369985.

E-mail address: mateo@iisaragon.es (R. Mateo-Gallego). 


\section{Introduction}

Obesity has become a major global health problem because it reduces life expectancy, entails an important socioeconomic impact, and is strongly associated with a number of comorbidities including metabolic syndrome, type 2 diabetes, and cardiovascular disease [1,2]. Overweight and obesity are associated with excess fat mass and adipocyte dysfunction [3]. The adipose tissue plays a key role in the regulation of energy metabolism and lipid and glucose blood homeostasis through its ability to secrete numerous proteins collectively known as adipokines [4,5]. The metabolic abnormalities induced by excess weight are highly associated with peripheral insulin resistance and excessive release of free fatty acids from adipocytes, and both these processes are considered markers of adipose tissue dysfunction [4,6,7]. Altered secretion of adipokines is a consequence of adipose tissue dysfunction and is a potent mediator of the inflammatory state and metabolic disorders associated with obesity [5-8].

Recent evidence showed that energy-restricted highprotein (HP) diets induce remarkable weight loss and result in a greater improvement of cardiometabolic parameters such as insulin sensitivity, plasma triglyceride, or HDL cholesterol levels when compared with carbohydratedense hypocaloric diets $[9,10]$. Hence, HP hypocaloric diets have been proposed as a potential approach in the treatment of type 2 diabetes [11,12]. In this line, our previous work demonstrated that an energy-restricted diet with $35 \%$ of calories from proteins more effectively improved the metabolic abnormalities associated with obesity in comparison with an energy-restricted diet that is low in protein content, independently of weight loss [10]. However, the mechanisms responsible for these differential effects of HP diets are unknown. Low-calorie HP diets tend to preserve fat-free mass and achieve higher fat mass reduction, and this mechanism has been proposed as a potential driver of their beneficial effects on lipids concentration or insulin resistance $[13,14]$. However, other studies could not confirm this mechanism as plausible [15]. Improvement in functionality rather than depletion in adipose tissue is a reasonable hypothesis that has been proposed too $[4,5]$. Some recently reviewed studies have described an increase in adiponectin and a decrease in RPB4 concentrations after weight loss, which have been related to glucose metabolism improvement [16]. However, few studies have explored the effect of HP diets on adipokine concentration by showing divergent effects among different cytokines. In addition to protein quantity, the source and quality of protein could play an essential role in its beneficial effects on metabolism. Animal protein consumption is associated with cardiometabolic parameter benefits, mainly in insulin resistance, blood pressure, or adiposity-related metabolites, among others [17-19].

Thus, we aimed to explore the relation between weight loss and plasma adipokine concentration associated with different adipose tissue functions and whether energyrestricted HP diets ( $80 \%$ of whom coming from lean animal protein) could induce further improvement in adipose tissue functionality beyond weight loss, which could be postulated as a mechanism responsible for the cardiometabolic benefits of HP diets associated with weight loss.

\section{Methods}

\section{Study population}

The study protocol has been previously described elsewhere [10]. Briefly, women were recruited to participate in a 3-month weight loss intervention study that was carried out at a University Hospital in northern Spain. Only women were selected to homogenize the study findings. Other inclusion criteria included age $18-80$ years, body mass index (BMI) $27.5-45 \mathrm{~kg} / \mathrm{m}^{2}$, and stable weight $( \pm 3 \mathrm{~kg}$ ) in the previous 3 months. The exclusion criteria included hypothyroidism, uncontrolled type-2 diabetes (glycated hemoglobin (HbA1c) > 8\%), any other disease that could interfere with the ability to comply with the study protocol, and current lipid-lowering or anti-diabetic drugs. Women taking supplements of phytosterols, omega-3 fatty acids, or any obesity drug were also excluded. Among participants meeting the study inclusion criteria, 91 women were randomly selected for randomization to one of three diets. A total of 80 women completed the 3-month dietary intervention period.

All subjects provided written informed consent to participate in the study. The study protocol was approved by the ethical committee of our institution (Comité de Ética e Investigación Clínica de Aragón); all procedures were in accordance with the ethical standards of that committee. This clinical trial was registered at ClinicalTrials.gov under identifier NCT02160496.

\section{Study design}

The study consisted of a 3-month weight loss intervention phase, which has been previously explained in detail [10]. We selected a 3-month period of time according to previous findings of weight loss effect on adipokine concentrations and considering that diet-induced cardiometabolic parameter changes stabilize in $<4$ weeks $[16,20]$. Intervention included individual consultations to reinforce messages and motivate weight loss. Clinical, anthropometric, dietary, and biochemical variables were assessed at baseline and after 3 months of dietary intervention.

The study consisted of a three-arm design, with subjects randomly assigned to one of three energy-reduced diets: $20 \%, 27 \%$, or $35 \%$ protein. The rest of macronutrients were distributed as follows: protein, $20 \%, 27 \%$, or $35 \%$; carbohydrates, $50 \%, 43 \%$, or $35 \%$, respectively; and fat, $30 \%$ in all diets. Once all screening visits were concluded, all subject data were recorded in a data file. The first woman to be included in the study was allocated to the $20 \%$-protein diet, the second to the $27 \%$-protein diet, the third to the $35 \%$-protein diet, and so on. Participants were blinded to their assigned macronutrient composition. Low-calorie diets involved a caloric restriction of $600 \mathrm{kcal}$ per day, which was applied to total daily energy expenditure energy intakes. They were 
estimated by multiplying the activity factor by resting energy expenditure calculated by Harris-Benedict equation. Approximately $80 \%$ of the protein came from lean animal sources, mainly lean lamb meat but also chicken or turkey, low-fat dairy products, etc. More information about diet composition and an example of a daily menu has been previously provided [10]. All participants were provided with physical activity advice that was in accordance with their physical status. Patients were counseled to increase exercise at each monitoring visit depending on the training reported at each visit to promote weight loss. Physical activity advice was quite heterogeneous because of varying fitness conditions of women (i.e., walking $1 \mathrm{~h}$ a day or running 30 min three times a week).

Dietary and physical activity assessments were performed at baseline and after 3 months of dietary intervention using a 3-day weighed food record and assessed according to the Spanish food composition tables and a validated questionnaire, respectively [21,22].

\section{Body weight and composition}

Anthropometric measurements (body weight and waist circumference) were evaluated at baseline (randomization) and after 3 months of dietary intervention. Body composition was assessed using bioelectrical impedance through the bipolar foot-to-foot technique (Tanita TBF 410 GS, Omron Corporation ${ }^{\circledR}$, Tokyo, Japan). Abdominal fat deposits were also measured using bioelectrical impedance (TanitaViScan AB-140, Omron Corporation ${ }^{\circledR}$ ) by evaluating visceral fat. Measurements were performed in the abdominal area with the patient in supine position with her hands on her chest. Abdominal fat composition was always determined at the navel, with an area $10 \mathrm{~cm}$ around it clear. As established by the manufacturer, abdominal visceral fat was expressed on a scale of $0-35$. All measurements were taken in accordance with the recommended guidelines: no food or drink $3 \mathrm{~h}$ prior to measurements, no exhausting exercise $12 \mathrm{~h}$ prior to measurements, and no alcohol or caffeine consumption $24 \mathrm{~h}$ prior to measurements [23].

\section{Clinical and laboratory parameters}

Blood pressure was measured in triplicate with a validated semiautomatic oscillometer (Omron M3, Omron Corporation, Hoofddorp, The Netherlands). Blood samples were drawn by venipuncture after $12 \mathrm{~h}$ of fasting at the randomization visit and at the 3-month visit. Levels of total cholesterol, triglycerides, HDL cholesterol, gammaglutamyl transpeptidase (GGT), and glutamic-pyruvic transaminase were measured using standard enzymatic methods. LDL cholesterol levels were estimated using the Friedewald formula when serum triglycerides were $<400 \mathrm{mg} / \mathrm{dL}$. Levels of non-HDL cholesterol were calculated as total cholesterol minus HDL cholesterol. We used the homeostasis model assessment-estimated insulin resistance (HOMA-IR) as a marker for insulin resistance. Blood glucose levels were measured by the glucose-oxidase method. Insulin levels were measured by radioimmunoassay. HOMA-IR was estimated as fasting serum glucose $(\mathrm{mg} / \mathrm{dL}) \times$ plasma insulin $(\mu \mathrm{U} / \mathrm{mL}) / 405$. HbA1c concentration was determined using high-performance liquid chromatography. C-reactive protein was determined by nephelometry using IMMAGE-Immunochemistry System (Beckman Coulter, USA).

\section{Adipokine determination}

We determined leptin, adiponectin, resistin, and retinolbinding protein 4 (RBP4) as relevant adipokines related to weight loss according to previous studies [16]. Adipokine profiles were determined using the Human Adipokine Magnetic Bead Panel 1 (adiponectin and resistin), Human Adipokine Magnetic Bead Panel 2 (leptin) and Human Kidney Injury Magnetic Bead Panel 6 (RBP4) protocols from the MILLIPLEX ${ }^{\circledR}$ MAP Kits (Cat. \#. HADK1MAG-61K, HADK2MAG-61K, HKI6MAG-99K, Millipore). Analyses were performed in duplicates, and plasma sample dilutions were done according to the detection range of each panel. Briefly, adipokine assay plates were washed with assay buffer in an orbital plate shaker for $10 \mathrm{~min}$ at room temperature. The assay buffer was decanted, and the standards, controls, and diluted plasma samples were mixed with the assay buffer in each well. After the addition of fluorescently labeled capture antibody-coated beads, plates were incubated overnight at $4{ }^{\circ} \mathrm{C}$ on an orbital shaker. After overnight incubation with capture antibodies to detect adiponectin, resistin, leptin, and RBP4, the well content was removed by washing as indicated in the instructions provided by the manufacturer. Biotinylated detection antibodies were then added to the wells and incubated for $1 \mathrm{~h}$ at room temperature while shaking. After incubation, streptavidin-phycoerythrin was added to each well, and plates were incubated for $30 \mathrm{~min}$ at room temperature with shaking. After this incubation period, samples were washed as previously described and resuspended in Sheath Fluid for 5 min. Plates were run on a LABSCAN 100 (Luminex), and data were collected and analyzed using the Luminex XPONENT ${ }^{\circledR}$ software.

\section{Statistical analyses}

We selected leptin change to calculate sample size because there are only four studies exploring HP diet effect on adipokine concentration, and only one reported significant differences in leptin change after dietary intervention [16]. Leptin variability was estimated as $78.5 \mathrm{ng} / \mathrm{mL}$, and we expected a difference in leptin change after a dietary intervention of $50 \mathrm{ng} / \mathrm{mL}$ among groups. A total sample size of 42 subjects per group was obtained by considering $90 \%$ power $(Z \beta$ unilateral $=1.282)$ to detect a between-treatment group difference and a confidence interval $(1-\alpha)$ of $95 \%$ ( $Z \alpha$ unilateral $=1.645)$. Continuous variables are expressed as mean \pm SD, mean (95\% confidence interval) or median (25th percentile-75th percentile) according to their distribution. Categorical variables are reported as percentages. ANOVA and Kruskal-Wallis tests were performed for the 
comparison of multiple independent variables. When applicable, post hoc adjusted comparisons were performed with the Bonferroni correction. Categorical variables were compared using the Pearson chi-squared test by including inter-group comparison. Pearson's and Spearman's tests of correlation were applied as appropriate. Differences in paired clinical and biochemical variables were calculated with the dependent t-test for paired samples or with the Wilcoxon test. To assess differences in adipokine concentration among diets and the effect of type of diet on different cardiometabolic parameter changes and the effect of RBP4 in this association, we applied multiple linear regression while including weight loss as an independent variable. All statistical analyses were performed with SPSS version 24.0 (SPSS Inc., Chicago, IL, USA), and significance was set at $P<0.05$.

\section{Results}

\section{Baseline characteristics and weight loss after dietary intervention}

Ninety-one women were enrolled into the study, of whom 80 completed it and attended at 3-month visit. Adipokine levels were determined in women with high dietary intervention compliance. High compliance was defined as protein consumption $\pm 3 \%$ of that prescribed, which was assessed by dietary analysis; four women were excluded (Fig. 1). Baseline clinical, biochemical, and dietary characteristics of 76 women in whom adipokine concentrations were analyzed are shown in Table 1 . There were no statistically significant differences at baseline between women assigned to each diet. Adipokine concentrations at baseline did not differ between women randomized to the different diets as shown in Table 2.

\section{Metabolic changes after dietary intervention}

Metabolic changes after weight loss in women in whom adipokine levels were analyzed are shown in Supplemental Table 1. They are quite similar to those that we observed in all women who were included in the study that has been previously published [10]. Systolic blood pressure, glucose, and HOMA significantly decreased within $27 \%$ - and 35\%-protein diets after dietary intervention. HDL cholesterol significantly decreased in $20 \%-$ and $35 \%$-protein diets, and we observed an improvement in lipid profile and GGT concentration only in the highest protein diet group. Fat mass, fat-free mass, and visceral fat reductions were noted in all groups. We observed statistical differences between diets in total cholesterol, triglycerides, and HDL cholesterol changes, noting the highest decreases in 35\%-protein diet group.

\section{Adipokine change after dietary intervention}

Weight loss after dietary intervention was $-9.24 \pm 3.60 \%$, $-9.93 \pm 5.20 \%$, and $-10.7 \pm 4.28 \%$ in $20 \%-, 27 \%$-, and $35 \%-$ protein diets, respectively, without statistically significant differences between diets $(P=0.56)$. Changes in adipokine concentrations after 3 months of dietary intervention and according to the diet group are shown in Table 2. RBP4 concentration significantly changed by $12.5 \%(-2.70,27.8)$,

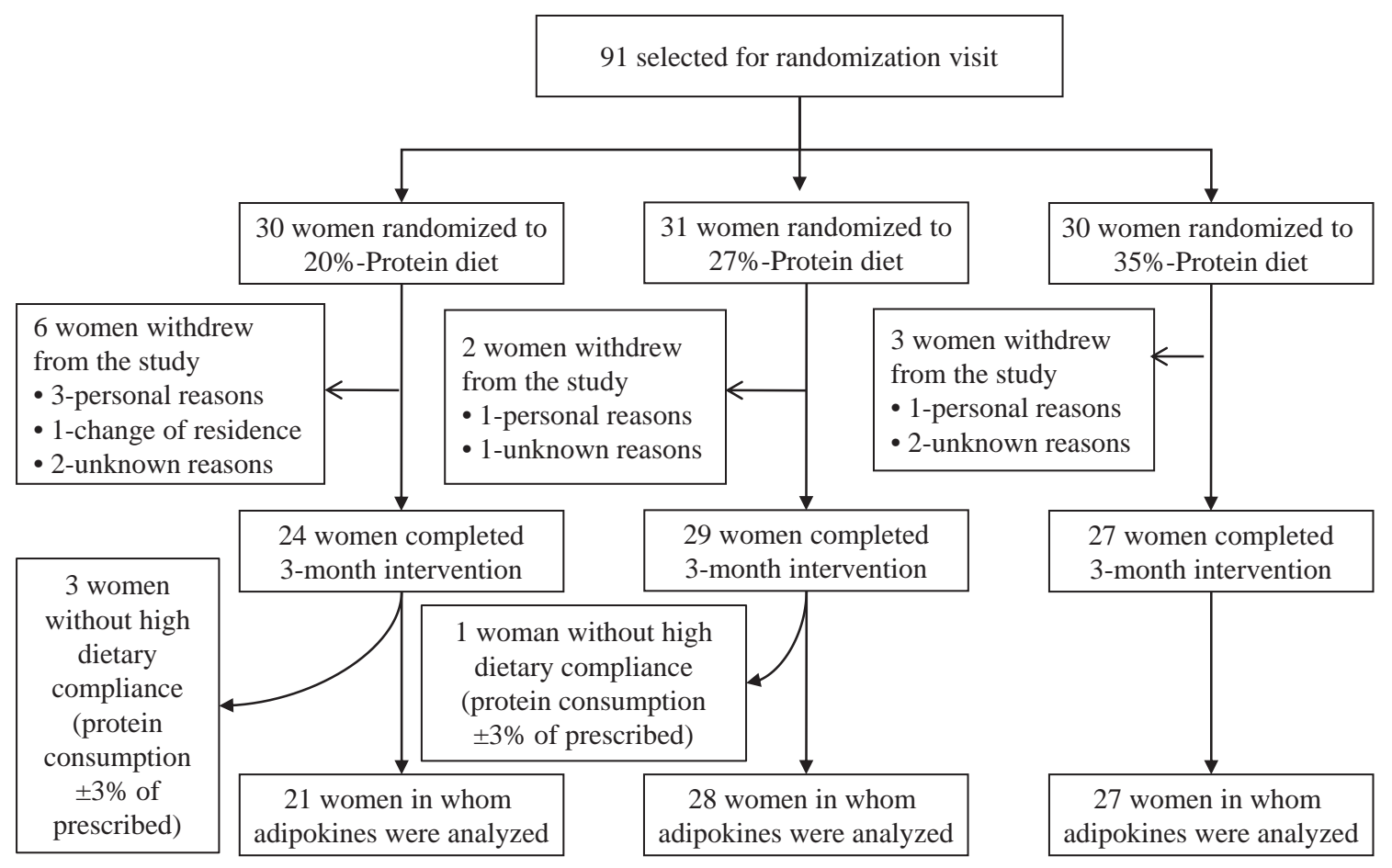

Figure 1 Flowchart of randomization and study course. 
Table 1 Baseline characteristics of participants according to diet group.

\begin{tabular}{|c|c|c|c|c|}
\hline & $\begin{array}{l}\text { 20\%-protein diet } \\
(\mathrm{N}=21)\end{array}$ & $\begin{array}{l}\text { 27\%-protein diet } \\
(\mathrm{N}=28)\end{array}$ & $\begin{array}{l}\text { 35\%-protein diet } \\
(\mathrm{N}=27)\end{array}$ & $P^{\mathrm{b}}$ \\
\hline Age, years & $43.7 \pm 9.74$ & $45.1 \pm 8.52$ & $43.2 \pm 9.17$ & 0.70 \\
\hline Weight, kg & $86.1 \pm 8.68$ & $88.3 \pm 9.40$ & $85.1 \pm 8.60$ & 0.41 \\
\hline BMI, $\mathrm{kg} / \mathrm{m}^{2}$ & $32.5 \pm 3.07$ & $33.7 \pm 2.68$ & $32.2 \pm 2.97$ & 0.16 \\
\hline Waist circumference, cm & $99.0 \pm 7.38$ & $100 \pm 9.30$ & $101 \pm 12.0$ & 0.82 \\
\hline Systolic blood pressure, mmHg & $124 \pm 12.0$ & $124 \pm 20.0$ & $119 \pm 13.1$ & 0.43 \\
\hline Diastolic blood pressure, $\mathrm{mmHg}$ & $77.7 \pm 9.23$ & $82.1 \pm 11.5$ & $79.6 \pm 9.76$ & 0.32 \\
\hline Total cholesterol, mg/dL & $208 \pm 40.0$ & $222 \pm 27.0$ & $212 \pm 34.0$ & 0.34 \\
\hline HDL cholesterol, mg/dL & $54.3 \pm 11.1$ & $55.9 \pm 9.28$ & $58.0 \pm 11.9$ & 0.49 \\
\hline Triglycerides, mg/dL & $98.0[72.0-128]$ & 113 [82.3-154] & 114 [92.0-149] & 0.39 \\
\hline LDL cholesterol, mg/dL & $127 \pm 45.5$ & $140 \pm 22.8$ & $129 \pm 26.6$ & 0.35 \\
\hline Non-HDL cholesterol, mg/dL & $154 \pm 35.3$ & $163 \pm 23.6$ & $154 \pm 27.5$ & 0.41 \\
\hline Glucose, mg/dL & $91.4 \pm 11.0$ & $90.6 \pm 15.3$ & $85.9 \pm 8.54$ & 0.22 \\
\hline HOMA-IR & 39.0 [24.5-72.2] & $38.9[30.9-82.1]$ & $39.3[25.1-53.1]$ & 0.59 \\
\hline HbA1c, \% & $5.46 \pm 0.31$ & $5.42 \pm 0.40$ & $5.42 \pm 0.29$ & 0.90 \\
\hline GGT, U/L & $18.0[13.0-27.5]$ & $20.0[16.0-31.0]$ & $20.0[14.0-30.0]$ & 0.38 \\
\hline ALT, U/L & $18.0[13.0-24.0]$ & $16.0[12.0-25.8]$ & $15.0[13.0-21.0]$ & 0.83 \\
\hline CRP, $\mathrm{g} / \mathrm{L}$ & $4.55[1.68-7.33]$ & $3.60[1.50-8.20]$ & $4.20[1.85-8.60]$ & 0.67 \\
\hline Fat mass, kg & $34.2 \pm 6.61$ & $36.0 \pm 7.06$ & $35.6 \pm 7.23$ & 0.65 \\
\hline Fat free mass, kg & $46.7 \pm 5.85$ & $48.2 \pm 4.12$ & $46.3 \pm 2.97$ & 0.26 \\
\hline Visceral fat, level & $9.10 \pm 1.79$ & $9.71 \pm 2.12$ & $9.22 \pm 2.28$ & 0.54 \\
\hline Physical activity level, METs/min & $954[371-1400]$ & $693[479-1328]$ & $693[480-1386]$ & 0.89 \\
\hline \multicolumn{5}{|l|}{ Dietary intake } \\
\hline Energy, kcal & $1925 \pm 428$ & $1804 \pm 438$ & $1893 \pm 416$ & 0.60 \\
\hline Protein, \% & $21.0[17.3-23.2]$ & $21.6[17.5-23.8]$ & $19.4[16.9-23.0]$ & 0.58 \\
\hline Animal protein, \% & $15.0[12.4-19.2]$ & $17.3[13.3-20.4]$ & $15.1[11.1-18.7]$ & 0.43 \\
\hline Vegetal protein, \% & $4.29[3.86-5.47]$ & $4.36[3.62-4.89]$ & $4.90[3.82-5.77]$ & 0.91 \\
\hline Total Fat, \% & $44.9 \pm 7.04$ & $44.7 \pm 5.70$ & $43.8 \pm 5.37$ & 0.78 \\
\hline Monounsaturated fat, \% & $21.1 \pm 3.44$ & $20.4 \pm 3.50$ & $19.9 \pm 3.56$ & 0.52 \\
\hline Polyunsaturated fat, \% & 6.18 [5.25-7.70] & $5.77[5.00-7.56]$ & $5.96[5.29-7.18]$ & 0.64 \\
\hline Saturated fat, \% & 13.3 [10.8-16.2] & $13.6[12.0-16.2]$ & $14.0[11.3-16.0]$ & 0.55 \\
\hline Carbohydrates, \% & $34.4[29.0-38.7]$ & $33.9[27.3-37.3]$ & $36.3[30.1-43.9]$ & 0.34 \\
\hline Sugars, g & 66.1 [54.6-79.0] & $64.4[52.1-84.8]$ & 73.7 [58.1-102] & 0.96 \\
\hline Fiber, $g$ & $18.7 \pm 9.16$ & $16.3 \pm 5.62$ & $17.9 \pm 6.43$ & 0.49 \\
\hline Alcohol, g & $0[0-0]$ & $0[0-1.68]$ & $0[0-0.57]$ & 0.58 \\
\hline
\end{tabular}

$3.56 \%(-9.87,17.0)$, and $-17.5 \%(-31.7,-3.22)$ in $20 \%-, 27 \%-$, and $35 \%$-protein diets, respectively $(P=0.008)$. We observed statistically significant differences when we compared the 35\%-protein diet with $20 \%$ - and $27 \%$-protein diets $(P$ for trend $=0.004)$. Women following the $35 \%$ protein diet showed 30\% greater decrease in RBP4 than those following the 20\%-protein $\operatorname{diet}(P=0.005 ; P=0.032$ after adjusting for weight loss). Leptin levels significantly decreased by $-43.0 \%(-55.3,-30.7),-36.8 \%(-47.1,-26.1)$, and $-50.3 \%(-61.2,-39.5)$ in $20 \%-, 27 \%-$, and $35 \%$-protein diets, respectively, without differences between diets. Adiponectin concentrations changed from basal concentration by $-0.60 \%(-15.8,14.6), 8.20 \%(-4.93,21.3)$, and $-12.9 \%$ $(-26.2,0.51)$ in $20 \%-, 27 \%$-, and $35 \%$-protein diets, respectively $(P$ for trend $=0.165$ ). Changes in resistin did not show statistically significant differences during the trial.

\section{Association between adipokine change and weight loss}

Leptin reduction showed a positive and significant correlation $(\mathrm{R}=0.59, \mathrm{P}<0.001)$ with weight loss (Supplemental
Table 2). Weight loss resulted in a 33.3\% reduction in leptin levels (B = 3.75; 95\% CI: 2.53, 4.97; $P<0.001$ ). RBP4 levels were slightly correlated with weight loss, although it was not statistically significant $(\mathrm{R}=0.22, P=0.069)$. Adiponectin and resistin levels were not significantly correlated with weight loss.

Fat mass and visceral fat change significantly were correlated with leptin variation (Supplemental Table 2). We observed a significant correlation between RBP4 levels and fat mass changes ( $R=0.34, P=0.003$ ). No significant correlations were observed between adipokine concentrations and fat-free mass changes.

\section{Association between adipokines and metabolic changes}

Crude correlations between adipokine concentration and metabolic changes are shown in Supplemental Table 2. Leptin variation was directly correlated with HOMA and HDL cholesterol change, adiponectin variation directly correlated with HDL cholesterol change, and RBP4 variation directly correlated with triglycerides concentration change. 
Table 2 Adipokine concentration changes after 3 months of intervention according to type of diet. ${ }^{\text {a }}$

\begin{tabular}{|c|c|c|c|c|c|c|c|c|c|c|}
\hline & & & \multicolumn{2}{|l|}{$20 \%$-protein diet } & \multicolumn{2}{|l|}{$27 \%$-protein diet } & \multicolumn{2}{|l|}{$35 \%$-protein diet } & \multirow[t]{2}{*}{ Overall $P^{\mathrm{b}}$} & \multirow[t]{2}{*}{$P$ for trend } \\
\hline & & & Mean (CI) & $P$ & Mean $(\mathrm{CI})$ & $P$ & Mean $(\mathrm{CI})$ & $P$ & & \\
\hline \multirow[t]{6}{*}{ RBP4 } & & Baseline, $10^{3}$ & $29.0(23.1,34.8)$ & - & $25.3(20.2,30.5)$ & - & $31.4(26.1,36.8)$ & - & 0.261 & - \\
\hline & & Average change & $12.5(-2.70,27.8)$ & - & $3.56(-9.87,17.0)$ & - & $-17.5(-31.7,-3.22)$ & - & 0.008 & \\
\hline & \multirow[t]{2}{*}{ Model 1: Raw } & Difference vs. $20 \%$ & Ref. & - & $-8.97(-29.3,11.3)$ & 0.381 & $-30.0(-50.9,-9.14)$ & 0.005 & 0.015 & 0.004 \\
\hline & & Difference vs. $35 \%$ & $30.0(9.14,50.9)$ & 0.005 & $21.0(1.45,40.6)$ & 0.036 & Ref. & - & & \\
\hline & \multirow{4}{*}{$\begin{array}{l}\text { Model 2: Adjusted for } \\
\text { weight loss }\end{array}$} & Difference vs. 20\% & Ref. & - & $-3.92(-23.7,15.8)$ & 0.693 & $-22.5(-43.1,1.94)$ & 0.032 & 0.033 & 0.027 \\
\hline & & Difference vs. $35 \%$ & $22.5(1.94,43.1)$ & 0.032 & $18.6(-0.39,37.6)$ & 0.055 & Ref. & - & & \\
\hline \multirow[t]{6}{*}{ Leptin } & & Baseline, $10^{6}$ & $27.1(21.2,32.9)$ & - & $28.2(23.1,33.2)$ & - & $28.4(23.3,33.6)$ & - & 0.937 & - \\
\hline & & Average change & $-43.0(-55.3,-30.7)$ & - & $-36.8(-47.1,-26.1)$ & - & $-50.3(-61.2,-39.5)$ & - & 0.210 & \\
\hline & \multirow{2}{*}{ Model 1: Raw } & Difference vs. $20 \%$ & Ref. & - & $6.56(-10.2,-22.5)$ & 0.446 & $-7.34(-23.7,-9.07)$ & 0.376 & 0.211 & 0.294 \\
\hline & & Difference vs. $35 \%$ & $7.34(-9.06,23.7)$ & 0.376 & $13.6(-1.62,28.8)$ & 0.079 & Ref. & - & & \\
\hline & \multirow{4}{*}{$\begin{array}{l}\text { Model 2: Adjusted for } \\
\text { weight loss }\end{array}$} & Difference vs. $20 \%$ & Ref. & - & $7.45(-6.15,21.04)$ & 0.278 & $-2.85(-16.8,11,1)$ & 0.684 & $<0.001$ & 0.553 \\
\hline & & Difference vs. $35 \%$ & $2.85(-11.1,16.8)$ & 0.684 & $10.3(-2.35,23.0)$ & 0.109 & Ref. & - & & \\
\hline \multirow[t]{6}{*}{ Adiponectin } & & Baseline, $10^{3}$ & $13.7(10.5,16.8)$ & - & $14.9(12.2,17.6)$ & - & $13.3(10.4,16.1)$ & - & 0.684 & - \\
\hline & & Average change & $-0.60(-15.8,14.6)$ & - & $8.20(-4.93,21.3)$ & - & $-12.9(-26.2,0.51)$ & - & 0.048 & \\
\hline & \multirow[t]{2}{*}{ Model 1: Raw } & Difference vs. $20 \%$ & Ref. & - & $8.79(-11.3,28.9)$ & 0.028 & $-12.3(-32.5,7.95)$ & 0.230 & 0.087 & 0.165 \\
\hline & & Difference vs. $35 \%$ & $12.3(-7.95,32.5)$ & 0.230 & $21.06(2.32,39.8)$ & 0.385 & Ref. & - & & \\
\hline & \multirow{4}{*}{$\begin{array}{l}\text { Model 2: Adjusted for } \\
\text { weight loss }\end{array}$} & Difference vs. $20 \%$ & Ref. & - & $8.52(-12.2,29.2)$ & 0.414 & $-11.7(-32.8,9.45)$ & 0.274 & 0.223 & 0.200 \\
\hline & & Difference vs. $35 \%$ & $11.7(-9.45,32.8)$ & 0.274 & $20.2(0.95,39.5)$ & 0.040 & Ref. & - & & \\
\hline \multirow[t]{6}{*}{ Resistin } & & Baseline, $10^{3}$ & $76.4(51.9,101)$ & - & $58.2(37.0,79.4)$ & - & $69.3(47.7,90.9)$ & - & 0.521 & - \\
\hline & & Average change & $2.64(-8.17,13.5)$ & - & $5.86(-3.51,15.2)$ & - & $4.01(-5.53,13.5)$ & - & 0.161 & \\
\hline & \multirow[t]{2}{*}{ Model 1: Raw } & Difference vs. $20 \%$ & Ref. & - & $3.22(-11.1,17.5)$ & 0.783 & $1.37(-13.1,15.8)$ & 0.850 & 0.902 & 0.889 \\
\hline & & Difference vs. $35 \%$ & $-1.37(-15.8,13.1)$ & 0.850 & $1.85(-11.5,15.2)$ & 0.655 & Ref. & - & & \\
\hline & \multirow{2}{*}{$\begin{array}{l}\text { Model 2: Adjusted for } \\
\text { weight loss }\end{array}$} & Difference vs. $20 \%$ & Ref. & & $4.63(-9.71,19.0)$ & 0.521 & $2.57(-12.1,17.2)$ & 0.728 & 0.478 & 0.778 \\
\hline & & Difference vs. $35 \%$ & $-2.57(-17.2,12.1)$ & 0.728 & $2.07(-11.3,15.4)$ & 0.759 & Ref. & & & \\
\hline
\end{tabular}

${ }^{a}$ Values are means (95\% confidence interval). Baseline adipokine values are expressed as $10^{3}$, except for adiponectin, which expressed as $10^{6}$.

Average change refers to \% change with respect to baseline.

Overall $P$ refers to differences between three diets calculated by ANOVA. 
Because of the differences in RBP4 variations between diets, we explored the potential impact of RBP4 variation on triglyceride change between them (Table 3). Women following the 35\%-protein diet showed a greater decrease in triglyceride concentration even when controlled for weight loss ( $\mathrm{B}=-32.5$; 95\% CI: $-54.8,10.1, P=0.005)$, RBP4 variation explained $24.9 \%((17.6-13.2) / 17.6)$ and $25.9 \%$ ((32.5-24.0)/32.5) of the effect on triglycerides change of $27 \%$ - and 35\%-protein diets with respect to 20\%-protein diet (Fig. 2). We did not find a significant effect of RBP4 on other cardiometabolic parameter changes between different diets (Supplemental Table 3).

\section{Discussion}

This study explores for the first time the effect of different energy-reduced HP diets, mainly coming from lean animal protein, on adipose tissue functionality measured through plasma adipokine concentration and its association with changes in lipid and glucose metabolism. In our work, a 35\%protein diet energy-restricted induced greater RBP4 improvement than energy-reduced diets with lower protein content regardless of weight loss; and this improvement are associated with enhanced triglycerides independently of weight loss.

The adipose tissue is composed of adipocytes and multiple non-adipocyte cells; including immune cells, especially macrophages that represent up to $50 \%$ of total cells in the presence of obesity [24]. Complex interactions among adipose tissue cells are key factors in multiple metabolic pathways, including regulation of energy metabolism, satiety, food behavior, and systemic inflammation. Many of the endocrine and paracrine actions of adipose tissue are the response to adipokines secretion. Up to 600 different adipokines have been described, many of them with unknown functions [4]. In this study, we have analyzed four different adipokines associated with four differential functions of adipose tissue $[4,16]$. Leptin has a central role in complex feeding behavior, appetite control, and food intake. It acts through the central nervous system activating proopiomelanocortin neurons and inhibiting neuropeptide $\mathrm{Y}$ and agouti-related peptide (AgRP) neurons. Resistin has primarily a peripheral mechanism promoting endothelial cell activation, smooth muscle cell proliferation, and increasing peripheral sympathetic nerve activity. Consequently, high levels of resistin have been associated with high blood pressure and risk of atherosclerosis in obese subjects [25]. Adiponectin is mainly produced in mature adipocytes and it is their most abundantly produced protein. Although with ubiquitous actions, adiponectin stimulates fatty acid transport and oxidation in muscle by promoting the expression of several genes involved in mitochondrial $\beta$-oxidation and is regulated by PPAR- $\alpha$ (PPAR- $\alpha$ - Peroxisome proliferatoractivated receptor alpha) [26]. Adiponectin increases fat combustion and energy utilization and is inversely associated with plasma triglyceride and positively associated with HDL cholesterol and insulin sensitivity [27]. RBP4 is believed to be directly involved in the pathogenesis of i-

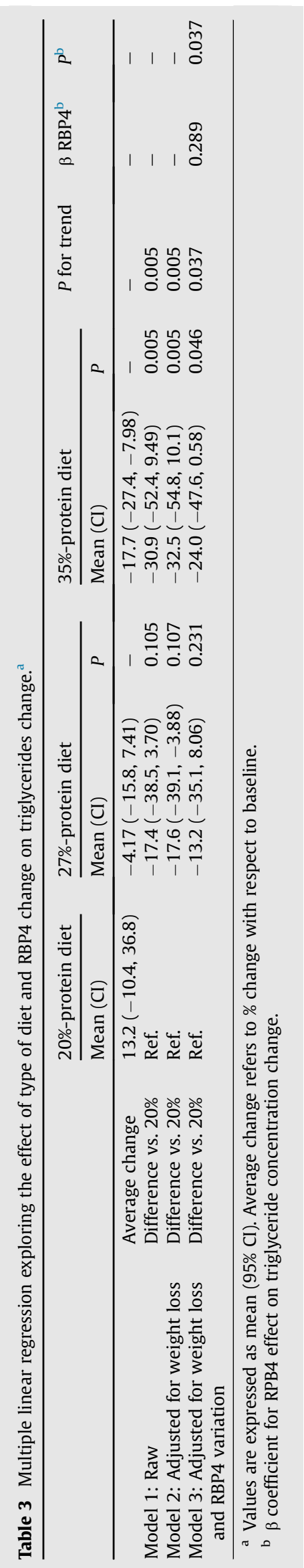




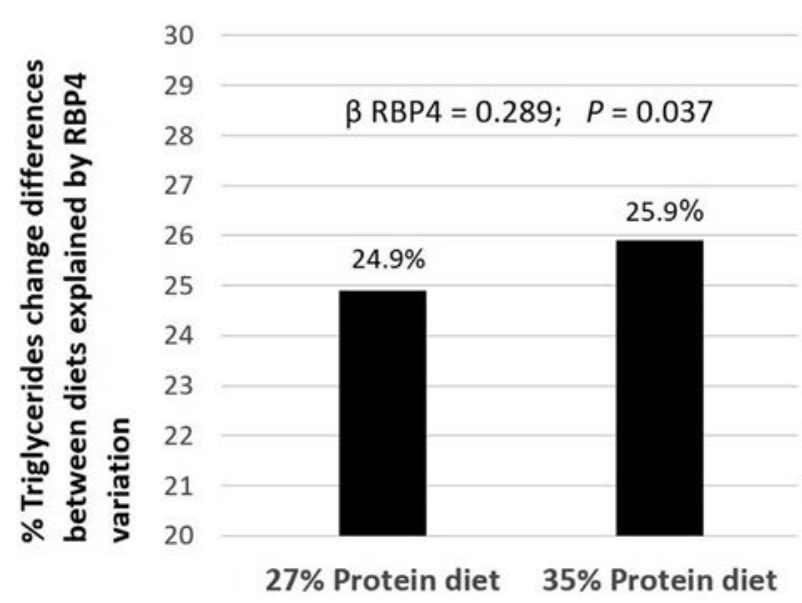

Figure $2 \%$ Triglyceride change differences between diets explained by RBP4 variation. $\beta$ coefficient and $P$ value for RBP4 change effect on triglyceride variation after adjusting for weight loss.

nsulin resistance. Transgenic overexpression of RBP4 in normal mice impairs muscle insulin signaling, and there is an association between RBP4 concentration and insulin resistance in humans [28].

In our study, leptin decreased similarly in all diets and was directly and significantly associated with weight loss, suggesting this adipokine as a good biomarker of mass adipose tissue. In fact, the primary source of leptin production is the mature adipocytes, and the intracellular fat content of adipocytes highly correlates with leptin gene expression [16,29]. According to the absence of differences in the leptin levels among diets, the results of the performed questionnaire including appetite, satiety, and food behavior did not differ among groups, as previously reported [10]. It is well known that even a small weight loss induced by caloric restriction has beneficial effects on leptin concentration. Reductions $<5 \%$ of total body weight decrease circulating levels of leptin in overweight and obese individuals although the best improvement is observed when weight loss is over $10 \%$ [16]. A study performed in 90 obesity non-diabetic outpatients showed decreases of $3.3 \%$ and $4.4 \%$ of weight loss that were associated to a reduction of leptin concentrations of $16.4 \%$ and $22.8 \%$ [30]. Another study observed in 48 healthy obese subjects a leptin reduction of $56 \%$ with a weight loss of $12 \%$ [31]. We observed similar reductions of $-43.0 \%,-36.8 \%$, and $-50.3 \%$ in $20 \%, 27 \%$, and $35 \%$ protein diets that achieved $-9.24 \%,-9.93 \%$, and $-10.7 \%$ weight loss, respectively. Our findings are consistent with those that positively associated the directly proportional expression and secretion of leptin to weight and fat mass suggesting a great biomarker of the extent of adipose tissue independently of diet composition obtaining the weight loss [32].

Adiponectin exhibits anti-atherogenic, insulin-sensitizing, and anti-inflammatory properties $[4,8]$. Some authors have described an increase in its concentration after weigh loss although others have not found any significant change [16]. Only one study has shown a decrease around $6 \%$ in its concentration in patients losing $8 \%-9 \%$ of body weight in a 12-week intervention study [33]. We have observed that adiponectin heterogeneously changed after weight loss with a reduction in 35\%-protein. No correlation with weight loss was denoted. The reason for these conflicting findings is not clear. It has been suggested that duration and calorie restriction could play an essential role in the findings, so further investigation is needed [16].

Few studies have specifically examined the effect of calorie-reduction intervention in RBP4 concentrations in humans. RBP4 concentration is increased and correlated with BMI in obese subjects, and weight reduction decreases RBP4 concentration [34-36]. However, the association between RBP4 and metabolic changes in relation with diet composition was not previously reported. We observed a significant reduction of $17.5 \%$ only in those women following the $35 \%$-protein diet. In our study, the change in RBP4 concentration did not correlate with weight loss in any group and the RBP4 reduction was independent of the amount of weight loss. There is limited information on the mechanisms involved in RBP4 expression and its association with adiposity and insulin resistance in humans [37]. Clinical studies support the hypothesis that RBP4 has a relevant role in obesity and the development of insulin resistance. HP diets have demonstrated to lead to cardiometabolic benefits mainly in insulin resistance. Protein source seems to play an essential role in these findings because animal protein has recently showed greater improvements in glucose and lipid metabolism and other cardiometabolic parameters [17-19]. Our $35 \%$-protein diet was enriched with lean meat, high in isoleucine and leucine, and therefore, RBP4 changes could be a potential mechanism explaining the benefits of diets with HP content. Furthermore, RBP4 is highly expressed not only in adipose tissue but also in the liver [38]. RBP4 is the specific transport protein for retinol in blood, and retinol is highly stored and catabolized to retinoic acid isomers in the liver, which act as ligands for multiple nuclear receptors to regulate transcription of hundreds of genes including many associated with glucose and lipid metabolism $[39,40]$. Hence, the mechanism involved in the greater reduction of RBP4 shown in the highest protein diet is probably related to changes in RBP4 expression by adipose tissue or the liver. The absence of differences in adipose tissue-origin adipokine changes between diets in our study suggests that the liver could be the origin of the variations in RBP4 levels observed with a 35\%-protein diet.

Our study has some limitations worth mentioning. The short intervention time could have influenced the findings in adipokine changes, such as adiponectin, which has been reported to be influenced by long-term weight loss exposure [16]. However, most interventional studies that have explored the effects of different weight loss interventions have got a similar time frame, so the findings are comparable. Diet compliance and physical activity were assessed by self-reported questionnaires, which is a limitation. More objective methods such as urine microalbumin and nitrogen concentration determination could have provided a more accurate assessment. Our study only involves women, so the findings and conclusions could not be extrapolated to other populations, and further research would be needed to 
confirm them. We have assessed adipose tissue functionality through adipokine concentration, which is well established [3-5]. However, future studies including in vivo experiments should confirm this hypothesis.

\section{Conclusion}

In conclusion, our study indicated that weight loss induced substantial changes in adipokines, which suggests improvement in adipose tissue functionality. However, only the highest protein diet induced a modification in RBP4 regardless of weight loss. This was directly associated with triglyceride concentration improvement. These findings suggest that HP diets improve the cardiometabolic profile, at least in part, through changes in adipokine secretion. Whether this beneficial effect of HP diet is due to improvements in hepatic or adipose tissue functionality could not be determined in our study. A high content of lean animal protein in diet could have played an essential role, which should be explored in future studies to fully elucidate the mechanisms and determine the optimal diet composition to optimize the clinical benefits of weight loss.

\section{Financial support}

This study was supported in part by three grants from the Carlos III Research Institute: CIBERCV (co-supported by the European Regional Development Fund (ERDF), which is allocated by the European Union; IIS16/0114), PI13/02507 and PI15/01983.

\section{Conflicts of interest}

None.

\section{Acknowledgments}

The authors thank the participants for their enthusiastic collaboration in the study and Antonio Oliván and Patricio Pérez from Pastores (Teruel, Spain) and I.G.P. Ternasco de Aragón (Zaragoza, Spain), respectively, for their help with study logistics. Martín Laclaustra research activity is funded by Agencia Aragonesa para la Investigación y el Desarrollo (ARAID).

\section{Appendix A. Supplementary data}

Supplementary data related to this article can be found at https://doi.org/10.1016/j.numecd.2017.10.024.

\section{References}

[1] Mozaffarian D. Dietary and policy priorities for cardiovascular disease, diabetes, and obesity: a comprehensive review. Circulation 2016;133:187-225.

[2] GBD 2015 Obesity Collaborators, Afshin A, Forouzanfar MH, Reitsma MB, Sur P, Estep K, Lee A, et al. Health effects of overweight and obesity in 195 countries over 25 years. N Engl J Med 2017;377:13-27.
[3] Blüher M. Adipose tissue dysfunction in obesity. Exp Clin Endocrinol Diabetes 2009; 117:241-50.

[4] Blüher M, Mantzoros CS. From leptin to other adipokines in health and disease: facts and expectations at the beginning of the 21st century. Metabolism 2015;64:131-45.

[5] Ouchi N, Parker JL, Lugus JJ, Walsh K. Adipokines in inflammation and metabolic disease. Nat Rev Immunol 2011;11:85-97.

[6] Ruscica M, Baragetti A, Catapano AL, Norata GD. Translating the biology of adipokines in atherosclerosis and cardiovascular diseases: gaps and open questions. Nutr Metab Cardiovasc Dis 2017; 27:379-95.

[7] Blüher M. Adipose tissue dysfunction contributes to obesity related metabolic diseases. Best Pract Res Clin Endocrinol Metab 2013;27:163-77.

[8] Chakraborti CK. Role of adiponectin and some other factors linking type 2 diabetes mellitus and obesity. World J Diabetes 2015;6: 1296-308.

[9] Wycherley TP, Moran LJ, Clifton PM, Noakes M, Brinkworth GD. Effects of energy-restricted high-protein, low-fat compared with standard-protein, low-fat diets: a meta-analysis of randomized controlled trials. Am J Clin Nutr 2012;96:1281-98.

[10] Mateo-Gallego R, Marco-Benedí V, Perez-Calahorra S, Bea AM, Baila-Rueda L, Lamiquiz-Moneo I, et al. Energy-restricted, highprotein diets more effectively impact cardiometabolic profile in overweight and obese women than lower-protein diets. Clin Nutr 2017;36:371-9.

[11] Pawlak R. Low-carbohydrate, high-protein diets for management of type 2 diabetes. Am J Clin Nutr 2013;98:247-8.

[12] American Diabetes Association. Standards of medical care in diabetes. Diabetes Care 2017:40:S1-2.

[13] Piatti PM, Monti F, Fermo I, Baruffaldi L, Nasser R, Santambrogio G, et al. Hypocaloric high protein diet improves glucose oxidation and spares lean body mass: comparison to hypocaloric highcarbohydrate diet. Metabolism 1994;43:1481-7.

[14] Leidy HJ, Carnell NS, Mattes RD, Campbell WW. Higher protein intake preserves lean mass and satiety with weight loss in preobese and obese women. Obesity (Silver Spring) 2007;15:421-9.

[15] Te Morenga L, Mann J. The role of high-protein diets in body weight management and health. Br J Nutr 2012;108(Suppl 2):S130-8.

[16] Klempel MC, Varady KA. Reliability of leptin, but not adiponectin, as a biomarker for diet-induced weight loss in humans. Nutr Rev 2011;69:145-54.

[17] Jennings A, MacGregor A, Pallister T, Spector T, Cassidy A. Associations between branched chain amino acid intake and biomarkers of adiposity and cardiometabolic health independent of genetic factors: a twin study. Int J Cardiol 2016;223:992-8.

[18] Berryman CE, Agarwal S, Lieberman HR, Fulgoni 3rd VL, Pasiakos SM. Diets higher in animal and plant protein are associated with lower adiposity and do not impair kidney function in US adults. Am J Clin Nutr 2016;104:743-9.

[19] Virtanen HEK, Koskinen TT, Voutilainen S, Mursu J, Tuomainen TP Kokko P, et al. Intake of different dietary proteins and risk of type 2 diabetes in men: the Kuopio Ischaemic Heart Disease Risk Factor Study. Br J Nutr 2017;117:882-93.

[20] Kris-Etherton PM, Dietschy J. Design criteria for studies examining individual fatty acid effects on cardiovascular disease risk factors: human and animal studies. Am J Clin Nutr 1997;65(Suppl): 1590S-6S.

[21] Mataix Verdú J, Mañas Almendros M. Tabla de composición de alimentos españoles (Spanish food composition tables). 4th ed. Granada, Spain: Universidad de Granada; 2003.

[22] Hagströmer M, Oja P, Sjostrom M. The International Physical Activity Questionnaire (IPAQ): a study of concurrent and construct validity. Public Health Nutr 2006;9:755-62.

[23] Thomas EL, Collins AL, McCarthy J, Fitzpatrick J, Durighel G, Goldstone AP, et al. Estimation of abdominal fat compartments by bioelectrical impedance: the validity of the ViScan measurement system in comparison with MRI. Eur J Clin Nutr 2010;64:525-33.

[24] Shoelson SE, Herrero L, Naaz A. Obesity, inflammation, and insulin resistance. Gastroenterology 2007;132:2169-80.

[25] Badoer E, Kosari S, Stebbing MJ. Resistin, an adipokine with nongeneralized actions on sympathetic nerve activity. Front Physiol 2015;6:321.

[26] Yoon MJ, Lee GY, Chung J-J, Ahn YH, Hong SH, Kim JB. Adiponectin increases fatty acid oxidation in skeletal muscle cells by sequential 
activation of AMP-activated protein kinase, p38 mitogen-activated protein kinase, and peroxisome proliferator-activated receptor $\alpha$. Diabetes 2006;55:2562-70.

[27] Ruan H, Dong LQ. Adiponectin signaling and function in insulin target tissues. J Mol Cell Biol 2016;8:101-9.

[28] Yang Q, Graham TE, Mody N, Preitner F, Peroni OD, Zabolotny JM, et al. Serum retinol binding protein 4 contributes to insulin resistance in obesity and type 2 diabetes. Nature 2005;436: 356-62.

[29] Maffei M, Halaas J, Ravussin E, Pratley RE, Lee GH, Zhang Y, et al. Leptin levels in human and rodent: measurement of plasma leptin and ob RNA in obese and weight-reduced subjects. Nat Med 1995; 1:1155-61.

[30] de Luis DA, Aller R, Izaola O, Gonzalez Sagrado M, Bellioo D, Conde R. Effects of a low-fat versus a low-carbohydrate diet on adipocytokines in obese adults. Horm Res 2007;67:296-300.

[31] Claessens M, van Baak MA, Monsheimer S, Saris WH. The effect of a low-fat, high-protein or high-carbohydrate ad libitum diet on weight loss maintenance and metabolic risk factors. Int J Obes (Lond) 2009;33:296-304.

[32] Wadden TA, Considine RV, Foster GD, Anderson DA, Sarwer DB, Caro JS. Short- and long-term changes in serum leptin in dieting obese women: effects of caloric restriction and weight loss. J Clin Endocrinol Metab 1998;83:214-8.

[33] Keogh JB, Brinkworth GD, Clifton PM. Effects of weight loss on a low-carbohydrate diet on flow-mediated dilatation, adhesion molecules and adiponectin. Br J Nutr 2007;98:852-9.
[34] Graham TE, Yang Q, Bluher M, Hammarstedt A, Ciaraldi TP, Henry RR, et al. Retinol-binding protein 4 and insulin resistance in lean, obese, and diabetic subjects. New Engl J Med 2006;354: 2552-63.

[35] Haider DG, Schindler K, Prager G, Bohdjalian A, Luger A, Wolzt M, et al. Serum retinol-binding protein 4 is reduced after weight loss in morbidly obese subjects. J Clin Endocrinol Metab 2007;92: 1168-71.

[36] Vitkova M, Klimcakova E, Kovacikova M, Valle C, Moro C, Polak J, et al. Plasma levels and adipose tissue messenger ribonucleic acid expression of retinol-binding protein 4 are reduced during calorie restriction in obese subjects but are not related to diet-induced changes in insulin sensitivity. J Clin Endocrinol Metab 2007;92: 2330-5.

[37] Kotnik P, Fischer-Posovszky P, Wabitsch M. RBP4: a controversial adipokine. Eur J Endocrinol 2011;165:703-11.

[38] Tsutsumi C, Okuno M, Tannous L, Piantedosi R, Allan M, Goodman DS, et al. Retinoids and retinoid-binding protein expression in rat adipocytes. J Biol Chem 1992;267:1805-10.

[39] Alapatt P, Guo F, Komanetsky SM, Wang S, Cai J, Sargsyan A, et al. Liver retinol transporter and receptor for serum retinol-binding protein (RBP4). J Biol Chem 2013;288:1250-65.

[40] Liu G, Ding M, Chiuve SE, Rimm EB, Franks PW, Meigs JB, et al, Plasma levels of fatty acid-binding protein 4, retinol-binding protein 4, high-molecular-weight adiponectin, and cardiovascular mortality among men with type 2 diabetes: a 22-year prospective study. Arterioscler Thromb Vasc Biol 2016;36:2259-67. 